CERN-TH/97-90

CPTH-S504.0497

IEM-FT-154/97

hep-th/9705037

\title{
Supersymmetry breaking in M-theory and gaugino condensation
}

\author{
I. Antoniadis ${ }^{a, b}$ and M. Quirós ${ }^{c}$ \\ ${ }^{a}$ TH-Division, CERN, CH-1211 Geneva 23, Switzerland \\ ${ }^{b}$ Centre de Physique Théorique, Ecole Polytechnique ${ }^{\dagger}$ F-91128 Palaiseau, France \\ ${ }^{c}$ Instituto de Estructura de la Materia, CSIC, Serrano 123, 28006 Madrid, Spain
}

\begin{abstract}
We argue that supersymmetry breaking by gaugino condensation in the strongly coupled heterotic string can be described by an analogue of Scherk-Schwarz compactification on the eleventh dimension in M-theory. The M-theory scale is identified with the gauge coupling unification mass, whereas the radius of the eleventh dimension $\rho$ is at an intermediate scale $\rho^{-1} \sim 10^{12} \mathrm{GeV}$. At the lowest order, supersymmetry is broken only in the gravitational and moduli sector at a scale $m_{3 / 2} \sim \rho^{-1}$, while it is mediated by gravitational interactions to the observable world. Computation of the mass splittings yields in general a hierarchy of soft masses at the TeV scale $\left(\sim \rho^{-2} / M_{p}\right)$ with matter scalars much heavier than gauginos.
\end{abstract}

${ }^{\star}$ Research supported in part by IN2P3-CICYT under contract Pth $96-3$, in part by CICYT contract AEN95-0195, and in part by the EEC under the TMR contract ERBFMRX-CT96-0090 and under CHRX-CT93-034.

${ }^{\dagger}$ Laboratoire Propre du CNRS UPR A.0014.

CERN-TH/97-90

April 1997 


\section{Introduction}

It is now widely believed that the strongly coupled $E_{8} \times E_{8}$ heterotic string theory is described by M-theory whose low energy limit is the eleven-dimensional supergravity [1]. In particular, four-dimensional (4D) $N=1$ supersymmetric vacua of the heterotic string compactified on Calabi-Yau (CY) manifolds are described, in the strong 10D coupling regime, by M-theory compactifications on $\mathrm{CY} \times S^{1} / Z_{2}$, the radius $\rho$ of the semicircle $S^{1} / Z_{2}$ being large [2]. In view of this duality, it is of the highest interest to discuss outstanding problems of perturbative string theory, such as supersymmetry breaking [3].

A first consequence of heterotic - M-theory duality is on gauge coupling unification [2, 4]. By identifying the M-theory scale with the unification mass $\sim 10^{16} \mathrm{GeV}$, it was noticed that the threshold of the eleventh dimension $\rho^{-1}$ is at an intermediate scale $\sim 10^{12} \mathrm{GeV}$, while the inverse size of the CY manifold (in the isotropic case) should also be of the order of the unification scale [4]. Furthermore, it was proposed to relate this intermediate scale with the scale of supersymmetry breaking. More precisely, when supersymmetry is broken on the M-theory side by a coordinate-dependent compactification analogue to the Scherk-Schwarz mechanism [5] using the eleventh dimension, one obtains that to lowest order only the gravitational sector is affected with a gravitino mass $m_{3 / 2} \sim \rho^{-1}$. The observable world, living at the end-points of the semicircle, remains unaffected and feels supersymmetry breaking only by gravitational interactions, which yield a hierarchy of values for the soft breaking terms in the TeV range, $m_{\text {susy }} \sim \rho^{-2} / M_{p}$. This therefore provides an appealing and economical scenario, which relates unification and supersymmetry breaking scales. Moreover, it was suggested that this situation could describe ordinary gaugino condensation in the strongly coupled heterotic string [四].

In this work, we make the above analogy with the heterotic string precise and perform a detailed analysis of supersymmetry breaking in M-theory. In section 2, we describe the compactification of M-theory on $\mathrm{CY} \times S^{1} / Z_{2}$ and discuss duality relations and the unification 
condition. In section 3, we review the main features of supersymmetry breaking by gaugino condensation in the heterotic string. Comparison with the duality results motivates the relation $m_{3 / 2} \sim 10^{12} \mathrm{GeV} \sim \rho^{-1}$, which can be obtained by a coordinate-dependent compactification on the eleventh dimension. This mechanism is made explicit in section 4 . In section 5, we compute the effects of supersymmetry breaking in the observable sector. We find that all scalar masses arise in general by gravitational interactions at the one-loop level as $\sim m_{3 / 2}^{2} / M_{p}$, while the dominant contributions to gaugino masses arise at the next level by gauge interactions as $\sim(\alpha / 2 \pi) m_{3 / 2}^{2} / M_{p}$. Finally, section 6 contains our conclusions and outlook.

\section{Compactification of M-theory on $\mathrm{CY} \times \mathrm{S}^{1} / \mathrm{Z}_{2}$}

Here, we review the main properties of M-theory compactification in four dimensions on a seven-dimensional internal space, which is the product of a Calabi-Yau manifold with the semicircle $S^{1} / Z_{2}$. Proceeding in two steps, we will first consider the five-dimensional theory on a Calabi-Yau threefold with Hodge numbers $h_{(1,1)}$ and $h_{(2,1)}$ leading to $N=15 \mathrm{D}$ spacetime supersymmetry [6]. In addition to the gravitational multiplet, the massless spectrum consists of $n_{V}=h_{(1,1)}-1$ vector multiplets and $n_{H}=h_{(2,1)}+1$ hypermultiplets. The gauge group is abelian, $U(1)^{n_{V}+1}$, where the additional factor counts the graviphoton. Starting with the eleven-dimensional bosonic fields, which are the metric $g$ and the 3-form gauge potential $A$, and splitting the Lorentz indices as $(\hat{\mu}, i, \bar{\jmath})$ with $\hat{\mu}=1, \ldots, 5$ and $i, \bar{\jmath}=1,2,3$, the $h_{(1,1)}$ gauge fields are given by $A_{\hat{\mu} i \bar{\jmath}}$ while the $h_{(1,1)}-1$ vector moduli correspond to $g_{i \bar{\jmath}}$ with unit determinant. Moreover, the hypermultiplet moduli are given by the $h_{(2,1)}$

complex scalar pairs $\left(g_{i j}, A_{i j \bar{k}}\right)$, along with the universal scalars $\left(\operatorname{det} g_{i \bar{\jmath}}, A_{\hat{\mu} \hat{\nu} \hat{\lambda}}, A_{i j k}=a \epsilon_{i j k}\right)$.

The second step consists in the compactification of the previous $5 \mathrm{D}$ theory down to four dimensions on $S^{1} / Z_{2}$, where the $Z_{2}$ acts as an inversion on the fifth coordinate $x_{5} \rightarrow-x_{5}$ and changes the sign of the 11D 3-form potential $A \rightarrow-A[1]$. As a result, one obtains 
$N=1$ supersymmetry in four dimensions together with $h_{(1,1)}+h_{(2,1)}+1$ massless chiral multiplets. The corresponding scalar moduli are the $h_{(1,1)}$ real pairs $\left(g_{i \bar{\jmath}}, A_{5 i \bar{\jmath}}\right)$, the $h_{(2,1)}$ complex scalars $g_{i j}$ and the universal real pair $\left(g_{55}, A_{5 \mu \nu}\right)$.

On top of the massless states, there is the usual tower of their Kaluza-Klein excitations with masses

$$
M^{2}=\frac{n^{2}}{\rho^{2}} \quad ; \quad n=0, \pm 1, \ldots
$$

corresponding to the fifth component of the momentum, $p_{5}$, which is quantized in units of the inverse radius of $S^{1}, 1 / \rho$. Because of the $Z_{2}$ projection, only the symmetric combination of their excitations $|n\rangle+|-n\rangle$ survive. On the other hand, the $Z_{2}$-odd states that were projected away at the massless level, give rise to massive excitations corresponding to the antisymmetric combination $|n\rangle-|-n\rangle$. It follows that all states of the 5D theory appear at the massive level.

In addition to these untwisted fields, there are twisted states located at the two endpoints of the semicircle, giving rise to the gauge group and to ordinary matter representations. In the case of the standard embedding, there is an $E_{8}$ sitting at one of the end-points and an $E_{6}$ with $h_{(1,1)} 27$ and $h_{(2,1)} \overline{\mathbf{2 7}}$ matter chiral multiplets sitting at the other. Of course, in any realistic model, $E_{6}$ should be further broken down to the Standard Model gauge group, for instance by turning on (discrete) Wilson lines.

This theory is believed to describe the strong coupling regime of the heterotic superstring compactified on the same Calabi-Yau manifold. The corresponding duality relations that express the eleven-dimensional parameters in terms of the four-dimensional ones are given by [2, 幽:

$$
\begin{aligned}
M_{11} & =\left(2 \alpha_{G} V\right)^{-1 / 6} \\
\rho^{-1} & =\left(\frac{2}{\alpha_{G}}\right)^{3 / 2} M_{p}^{-2} V^{-1 / 2}=\frac{4}{\alpha_{G}} M_{11}^{3} M_{p}^{-2}
\end{aligned}
$$

where $\alpha_{G}$ is the gauge coupling at the unification scale, $M_{p}=G_{N}^{-1 / 2}$ is the Planck mass, 
$(2 \pi)^{6} V$ is the volume of the Calabi-Yau space, and $M_{11}$ the mass scale of M-theory.

The description of the strongly coupled heterotic string in terms of M-theory is valid in the region where the ten-dimensional heterotic coupling is large, implying that $\rho$ is much

larger than the Planck length, or more precisely $\rho \gg 4 \alpha_{G}^{-1 / 2} M_{p}^{-1}$. This condition is held, in particular, when we identify the M-theory scale with the unification mass, $M_{11} \sim M_{G} \simeq$ $2 \times 10^{16} \mathrm{GeV}$, inferred from the low-energy data. Equation (2.2) then implies [4, (7]:

$$
\rho^{-1} \simeq 10^{12} \mathrm{GeV}
$$

while the compactification scale for an isotropic Calabi-Yau manifold is also of the order of $M_{G}, V^{-1 / 6} \simeq 1.3 \times 10^{16} \mathrm{GeV}$.

From the heterotic point of view, although the $4 \mathrm{D}$ coupling is small $\left(\alpha_{G} \sim 1 / 25\right)$ there are strong coupling effects associated to the fact that the $10 \mathrm{D}$ coupling is large, because the compactification scale $V^{-1 / 6}$ is smaller than the heterotic string scale $M_{H}=\left(\alpha_{G} / 8\right)^{1 / 2} M_{p} \sim$ $10^{18} \mathrm{GeV}[8]$. As a result, above the intermediate scale (2.3) the $4 \mathrm{D}$ effective field theory description breaks down, the "eleventh" dimension of the M-theory opens up, and the theory behaves effectively as five-dimensional. However, in this compactification, only the gravitational and moduli sectors of the theory live in the 5D bulk. The gauge and matter sectors live on the two 4D boundaries associated to the two end-points of the semicircle. The "observable" world lives on one of the boundaries while the "hidden" $E_{8}$ is sitting at the other. They communicate with each other and with the bulk only through gravitational interactions.

\section{Supersymmetry breaking in the heterotic string by gaugino condensation}

On the heterotic side, one expects that (local) supersymmetry can be broken by gaugino condensation effects in the hidden $E_{8}$, at least in the (10D) weakly coupled regime [9]. Let 
us describe briefly the main features of this mechanism. The physical picture is that the condensate $\langle\lambda \lambda\rangle$ develops at a scale $\Lambda_{c}$, where the gauge coupling of the hidden $E_{8}$ becomes strong:

$$
\langle\lambda \lambda\rangle \sim \Lambda_{c}^{3}=\mu^{3} e^{-\frac{2 \pi}{c_{8} \alpha 8}(\mu)}
$$

with $c_{8}=30$ being the quadratic Casimir of $E_{8}$ and $\alpha_{8}(\mu)$ its coupling constant at the scale $\mu$.

This phenomenon can be described by introducing a chiral supermultiplet $U$ whose vacuum expectation value (VEV) reproduces the condensate (3.1) [10]. The effective nonperturbative superpotential is determined by consideration of the anomalous Ward identities:

$$
W_{\mathrm{np}} \propto U\left(\frac{1}{\alpha_{W}}+\frac{c_{8}}{2 \pi} \ln \frac{U}{\mu^{3}}\right),
$$

where $\alpha_{W}$ is the Wilsonian effective coupling (at the scale $\mu$ ), which depends holomorphically on the moduli [11]. It is related to the physical coupling by:

$$
\frac{1}{\alpha_{8}}=\operatorname{Re} \frac{1}{\alpha_{W}}+\frac{c_{8}}{4 \pi}(-K+2 \ln (S+\bar{S}))
$$

where $K$ is the Kähler potential and $S$ is the heterotic dilaton whose VEV determines the $4 \mathrm{D}$ string coupling constant, $\operatorname{Re} S=1 / \alpha_{G}$.

Minimization of the effective potential with respect to $U$ implies to leading order in $\Lambda_{c} / M_{p}$ the condition $\partial_{U} W_{\mathrm{np}}=0$ [12], which gives

$$
U=\mu^{3} e^{-\frac{2 \pi}{c_{8} \alpha}-1} \quad ; \quad W_{\mathrm{np}} \propto U
$$

Using this result together with eqs. (3.1) and (3.3), it is straightforward to obtain the value of the gravitino mass:

$$
m_{3 / 2}=\left|W_{\mathrm{np}}\right| e^{K / 2} \propto \frac{1}{\alpha_{G}} \Lambda_{c}^{3} M_{p}^{-2}
$$

The effective potential should also be minimized with respect to the dilaton field $S$. Unfortunately, its runaway behaviour drives the theory to the supersymmetric limit with 
vanishing coupling, $S \rightarrow \infty$. A possible stabilization mechanism was initially proposed by means of a VEV for the field-strength of the antisymmetric tensor field along the compact directions, which shifts the superpotential by a constant [9]. However, this constant was found to be quantized, so that $W_{\mathrm{np}}$ becomes of order one at the minimum [13]. Then, eq. (3.5) implies that the only way to obtain a hierarchy for the gravitino mass is by making $e^{K / 2}$ small, or equivalently by having a large compactification volume $V \sim e^{-K}$. As a result, we obtain the following scaling relations (in $M_{p}$ units):

$$
m_{3 / 2} \sim V^{-1 / 2} \quad ; \quad \Lambda_{c} \sim V^{-1 / 6}
$$

Assuming that eqs. (3.5) and (3.6) hold in the strong coupling regime, a comparison with the duality relations (2.2) implies the identification of the condensation scale $\Lambda_{c}$ with the M-theory scale $M_{11}$ and the inverse radius of the semicircle $\rho^{-1}$ with the gravitino mass:

$$
\Lambda_{c} \sim M_{11} \quad ; \quad m_{3 / 2} \sim \rho^{-1}
$$

On the other hand, the scale $m_{3 / 2}$ can be fixed by imposing the unification condition $M_{11} \sim M_{G}$, which is also consistent with the fact that $\Lambda_{c}$ is very close to $M_{G}$, owing to the rapid variation of the $E_{8}$ gauge coupling. More precisely, taking $\Lambda_{c} \sim 10^{16} \mathrm{GeV}$, one obtains from eq. (3.5) $m_{3 / 2} \sim 10^{12} \mathrm{GeV}$, precisely the value (2.3) deduced from unification. This scenario can be phenomenologically viable if all mass splittings in the observable sector are zero to $\mathcal{O}\left(m_{3 / 2}\right)$ and are originated by gravitational interactions. Such a situation was realized in the past 114, 15, in the simplest case where the gaugino condensation potential is stabilized by the VEV of the antisymmetric tensor field-strength, because of the particular form of the effective field theory.

The previous arguments suggest that the gaugino condensation mechanism for supersymmetry breaking in the strongly coupled heterotic string could be described on the M-theory side by the field-theoretical Scherk-Schwarz mechanism on the eleventh dimension, which generically yields the desired relation $m_{3 / 2} \sim \rho^{-1}$. This is our main motivation 
to study supersymmetry breaking in M-theory by such coordinate-dependent compactification, as we describe in the next section.

\section{Supersymmetry breaking in M-theory by the Scherk-Schwarz mechanism on the eleventh dimension}

The Scherk-Schwarz mechanism of supersymmetry breaking makes use of a symmetry of the theory transforming the gravitino non-trivially. Upon compactification of the coordinate $x_{5}$ on a circle of radius $\rho$, one then chooses the five-dimensional fields to be periodic up to a symmetry transformation, $\Phi_{q}\left(x_{5}+2 \pi \rho\right)=e^{2 i \pi q \omega} \Phi_{q}\left(x_{5}\right), q$ being the charge and $\omega$ the transformation parameter. This boundary condition leads to a shift of the momentum $p_{5}$ along $x_{5}$ as $p_{5}=(n+q \omega) / \rho$, which gives rise to mass shifts in the four-dimensional theory by $q \omega / \rho$. Since the symmetries that transform the gravitino are usually discrete $R$-symmetries, the parameter $\omega$ is quantized and the scale of supersymmetry breaking is set by $\rho^{-1}$.

These symmetries are in fact discrete internal rotations left over after the compactification. Since in our case the coordinate $x_{5}$ is compactified on a semicircle $S^{1} / Z_{2}$, consistency of the theory requires that the corresponding Noether current must transform with a minus sign under the $Z_{2}$ orbifold action. This implies that the symmetry must be a rotation on a plane defined by $x_{5}$ and one of the internal Calabi-Yau coordinates. For a generic CalabiYau all such transformations are broken with the possible exception of a $2 \pi$ rotation. This

rotation acts on the $5 \mathrm{D}$ fields as the space-time parity $(-)^{2 s}$, which changes the sign of fermions and leaves bosons invariant. As a result, all fermions in the bulk receive a uniform shift of their $p_{5}$ momentum as $p_{5}=(n+1 / 2) / \rho$, while bosons remain untouched. On the other hand, twisted states living at the edges of the semicircle have no $p_{5}$ momenta and 
consequently no shifts. As a result, the general mass formula (2.1) is modified to:

$$
\begin{aligned}
M_{\text {ferm-bulk }}^{2} & =\frac{\left(n+\frac{1}{2}\right)^{2}}{\rho^{2}} \\
M_{\text {bos-bulk }}^{2} & =\frac{n^{2}}{\rho^{2}} \\
M_{\text {boundary }}^{2} & =0 .
\end{aligned}
$$

Equation (4.1) shows that supersymmetry is broken, since all supermultiplets in the bulk are split. In particular the $4 \mathrm{D}$ gravitino, as well as all fermion moduli get a common mass:

$$
m_{3 / 2}=\frac{1}{2 \rho}
$$

This result provides the desired relation, discussed in section 3, so as to describe gaugino condensation in the strong coupling regime of the heterotic string. Moreover, since the observable sector lives on the boundary, we obtain from eq. (4.1) vanishing mass splittings to $\mathcal{O}\left(m_{3 / 2}\right)$. Supersymmetry breaking will then be communicated from the bulk by gravitational interactions, as was the case of the gaugino condensation models mentioned in the previous section [14, 15.

The breaking (4.1) is identical to the one produced by finite temperature, where $\rho^{-1}$ is replaced by the temperature $T$, which plays the role of the inverse radius of the time coordinate. Since the tree-level scalar potential remains flat along the $\rho$-direction, the scale of supersymmetry breaking is classically undetermined. To satisfy the unification condition (2.3) we need the gravitino mass to be at the intermediate scale $\sim 10^{12} \mathrm{GeV}$. In the following, we will assume that this value is determined by some dynamical mechanism in the M-theory analogue to the gaugino condensation, and explore its consequences for supersymmetry breaking in the observable sector.

Note that the mass formula (4.1) is similar to the one obtained in the case of orbifold compactifications of the heterotic string, where $\rho$ is replaced by some large internal radius 
$R$ [16]-18]. One difference is the presence of the string winding modes whose inclusion is determined by modular invariance. Another substantial difference is the fact that part of the observable matter, including all gauge multiplets, belong to the untwisted (bulk) sector of the orbifold. Thus, their infinite tower of Kaluza-Klein excitations generically contributes to the renormalization of gauge couplings and lead to large threshold corrections, which break the validity of perturbation theory above the compactification scale $1 / R$. One then has to consider special models where this problem can be avoided [17]. In contrast, this problem is absent in the present case since only gravitationally coupled fields belong to the five-dimensional bulk.

\section{Communication of supersymmetry breaking to the observable sector}

As explained in the previous section, at the lowest order, supersymmetry is broken only in the five-dimensional bulk (gravitational and moduli sector), while it remains unbroken in the observable sector. The communication of supersymmetry breaking is then expected to arise radiatively, by gravitational interactions. This issue is studied below.

\section{Scalar masses}

At the one-loop level, the diagrams that contribute to the scalar masses in the observable sector are shown in Fig. 1, where the vertices come from the kinetic terms. Fields from the boundary, denoted by $\left(\varphi, \psi_{\varphi}\right)$, always appear in pairs, as dictated by the $Z_{2}$ invariance. Moreover, in the effective field theory limit $\rho M_{11} \gg 1$, their couplings to fields from the bulk are the same for all Kaluza-Klein excitations. The latter are the moduli $\left(z^{(n)}, \chi^{(n)}\right)$

and the graviton $\left(g_{\mu \nu}^{(n)}, \psi_{\mu}^{(n)}\right)$ supermultiplets. Moduli and matter field indices are dropped for notational simplicity. 
Every diagram from Fig. 1 gives a contribution proportional to:

$$
\begin{aligned}
I=\kappa^{2} \sum_{n} \int \frac{d^{4} k}{(2 \pi)^{4}} \frac{k^{2}}{k^{2}+M_{n}^{2}} & =\kappa^{2} \sum_{n} \int \frac{d^{4} k}{(2 \pi)^{4}} k^{2} \int_{0}^{\infty} d s e^{-s\left(k^{2}+M_{n}^{2}\right)} \\
& =\frac{1}{\pi M_{p}^{2}} \sum_{n} \int_{0}^{\infty} \frac{d s}{s^{3}} e^{-s M_{n}^{2}}
\end{aligned}
$$

where $\kappa=\sqrt{8 \pi} / M_{p}$ and the masses $M_{n}$ are given in eq. (4.1). After adding the contributions of diagrams related by supersymmetry, one finds:

$$
\begin{aligned}
I_{\mathrm{bos}}-I_{\mathrm{ferm}} & =\frac{1}{\pi M_{p}^{2}} \int_{0}^{\infty} \frac{d s}{s^{3}} \sum_{n}\left[e^{-s n^{2} / \rho^{2}}-e^{-s\left(n+\frac{1}{2}\right)^{2} / \rho^{2}}\right] \\
& =\frac{1}{\pi M_{p}^{2}} \int_{0}^{\infty} \frac{d s}{s^{3}}\left[\theta_{3}\left(\frac{i s}{\pi \rho^{2}}\right)-\theta_{2}\left(\frac{i s}{\pi \rho^{2}}\right)\right] \\
& =\frac{1}{\pi M_{p}^{2}} \int_{0}^{\infty} \frac{d s}{s^{3}}\left(\frac{\pi \rho^{2}}{s}\right)^{1 / 2}\left[\theta_{3}\left(\frac{i \pi \rho^{2}}{s}\right)-\theta_{4}\left(\frac{i \pi \rho^{2}}{s}\right)\right],
\end{aligned}
$$

where $\theta_{i}$ are the Jacobi theta-functions and in the second line of eq. (5.2) we have used the Poisson resummation formula. Note that the integral over $s$ is convergent both in the ultraviolet $(s=0)$ and in the infrared $(s=\infty)$ regions.

After rescaling the integration variable $s=x \rho^{2}$, and putting together all diagrams of Fig. 1, we obtain the following expression for the scalar masses:

$$
m_{\varphi \bar{\varphi}}^{2} \sim G_{\varphi \bar{\varphi}}^{-1}\left(G^{i \bar{\jmath}} R_{i \bar{\jmath} \varphi \bar{\varphi}}-G_{\varphi \bar{\varphi}}\right) \frac{m_{3 / 2}^{4}}{M_{p}^{2}} \mathcal{J},
$$

where we used the relation (4.2) for $m_{3 / 2}$, and $\mathcal{J}$ is a constant given by:

$$
\begin{aligned}
\mathcal{J} & =\int_{0}^{\infty} \frac{d x}{x^{3}}\left(\frac{\pi}{x}\right)^{1 / 2}\left[\theta_{3}\left(\frac{i \pi}{x}\right)-\theta_{4}\left(\frac{i \pi}{x}\right)\right] \\
& =\sqrt{\pi} \int_{0}^{\infty} d y y^{3 / 2} \sum_{n}\left[1-(-)^{n}\right] e^{-\pi^{2} n^{2} y} \\
& =\frac{93}{32 \pi^{4}} \zeta(5) \simeq(0.17)^{2},
\end{aligned}
$$

where $y=1 / x$ and $\zeta$ is the Riemann zeta-function. In eq. (5.3), $G_{i \bar{\jmath}}$ and $G_{\varphi \bar{\varphi}}$ are the moduli and matter metrics, while $R_{i \bar{\jmath} \varphi \bar{\varphi}}$ is the moduli-matter mixed Riemann tensor. The factor $G_{\varphi \bar{\varphi}}^{-1}$ comes from the wave function renormalization and the two terms in the bracket 
correspond to the contributions of the moduli and graviton supermultiplets, respectively, in the loops of Fig. 1. As a result, we find the scalar masses $m_{\varphi \bar{\varphi}}=\mathcal{O}\left(10^{-1}\right) m_{3 / 2}^{2} / M_{p} \sim$ $10^{4} \mathrm{GeV}$ generically. This is only a rough estimate, since besides the moduli dependent prefactor in eq.(5.3), the result is very sensitive to the value of $M_{11}$. In fact, eqs. (2.2) and (4.2) show that $m_{\varphi \bar{\varphi}}$ scales as $M_{11}^{6}$ and, thus, a factor of 2 in $M_{11}$ changes the scalar masses by almost two orders of magnitude.

Note that the scalar masses (5.3) turn out to be diagonal in the flavour space, if the matter metric is so. However, they need not be universal, owing to the presence of the Riemann tensor which in general can introduce a different moduli dependence for the various scalar fields. This may create dangerous flavour-changing neutral currents, a problem that is also shared by most string constructions and gives strong restrictions to model building.

A similar analysis can be applied to compute the masses of the scalar moduli. The corresponding diagrams are the same as those in Fig. 1, except that $\varphi$ is replaced, in the external lines, by the lowest excitation of the moduli $z^{(0)}$ and $\left(\varphi, \psi_{\varphi}\right)$ are replaced, in the internal lines, by the excitations $\left(z^{(-n)}, \chi^{(-n)}\right)$. Here, we use momentum conservation along the $x_{5}$-coordinate since the vertices involve only fields from the bulk and can therefore be obtained by $Z_{2}$ truncation of the dimensionally reduced theory. The evaluation of the diagrams can be done in the same way as before with the result:

$$
m_{z \bar{z}}^{2} \sim 5 G_{z \bar{z}}^{-1}\left(R_{z \bar{z}}-G_{z \bar{z}}\right) \frac{m_{3 / 2}^{4}}{M_{p}^{2}} \mathcal{J},
$$

where $R_{z \bar{z}}$ is the moduli Ricci tensor and the constant $\mathcal{J}$ is given in eq. (5.4). Thus, all moduli scalars obtain masses of the same order as the scalar masses in the observable sector $\mathcal{O}(10) \mathrm{TeV}$.

Although the moduli metric appearing in expressions (5.3) and (5.5) is in general arbitrary, it has special properties in the limit under consideration where the radius $\rho$ is large. In this limit, the theory behaves as five-dimensional with $N=2$ supersymmetry from the point of view of four dimensions. The moduli metric then becomes block-diagonal 
in the space of $N=2$ vector and hypermultiplets. Furthermore, in the large radius limit, the metric of the $h_{(1,1)}$ vector moduli is considerably simplified by the underlying $N=2$ special geometry with a cubic prepotential [6]. On the other hand, the metric of the remaining $h_{(2,1)}+1$ moduli, which can be obtained by a truncation of the corresponding quaternionic manifold, remains arbitrary in general. It would be interesting to further analyse the consequences of these properties in particular models [19].

The fact that all scalar squared mass splittings are of order $m_{3 / 2}^{4} / M_{p}^{2}$ is a consequence of the absence of quadratic divergences in the effective supergravity. Inspection of eq. (5.2) shows that cancellation of quadratic divergences arises non-trivially. Indeed, any single excitation $n$ of the sum gives a contribution to the integral, which is quadratically divergent at $s=0$ as $d s / s^{2}$, so that after introducing an ultraviolet cutoff $1 / M_{p}^{2}$ one would get a contribution of order $m_{3 / 2}$ to the mass. However, after summing over all modes and performing the Poisson resummation, one finds that the integrand has an exponentially suppressed (non-analytic) ultraviolet behaviour as $e^{-\pi^{2} \rho^{2} / s} / s^{5 / 2}$. This phenomenon is very similar to the situation at finite temperature upon the identification $T \equiv 2 m_{3 / 2}$, as was discussed in section 4. To make the analogy more explicit, one can also compute the effective potential as a function of the background $\rho$ :

$$
\begin{aligned}
V_{\text {eff }} & =\frac{1}{2} \operatorname{Str} \int \frac{d^{4} k}{(2 \pi)^{4}} \ln \left(k^{2}+M^{2}\right) \\
& =-\frac{N}{32 \pi^{2}} \int_{0}^{\infty} \frac{d s}{s^{3}} \sum_{n}\left[e^{-s n^{2} / \rho^{2}}-e^{-s\left(n+\frac{1}{2}\right)^{2} / \rho^{2}}\right] \\
& =-\frac{N \mathcal{J}}{32 \pi^{2}} \frac{1}{\rho^{4}}
\end{aligned}
$$

where $N$ is the number of massless multiplets from the bulk. This result corresponds to the well-known behaviour at finite temperature where $V_{\text {eff }} \propto T^{4}$ and shows the vanishing of $\operatorname{Str} M^{2}$ after supersymmetry breaking.

\footnotetext{
${ }^{1}$ We thank S. Dimopoulos for discussions on this point.
} 


\section{Gaugino masses}

The diagrams that contribute to the gaugino masses at the one-loop level are shown in Fig. 2, where the vertices again come from the kinetic terms. They lead to individual contributions proportional to:

$$
\kappa^{2} \sum_{n} \int \frac{d^{4} k}{(2 \pi)^{4}} \frac{\left|M_{n}\right|}{k^{2}+M_{n}^{2}} .
$$

The total contribution to the mass can then be written as:

$$
\begin{aligned}
m_{\lambda \lambda} & \propto \frac{1}{M_{p}^{2}} \int_{0}^{\infty} \frac{d s}{s^{5 / 2}} \sum_{n}\left[e^{-s n^{2} / \rho^{2}}-e^{-s\left(n+\frac{1}{2}\right)^{2} / \rho^{2}}\right] \\
& \sim \frac{m_{3 / 2}^{3}}{M_{p}^{2}}
\end{aligned}
$$

where we followed the same steps as in the case of scalar masses.

The above result shows that the one-loop gravitational contributions to gaugino masses are too small for phenomenological purposes. This is a general problem, which has been known for a long time [14, 15]. A possible solution exists if there are massive matter fields transforming non-trivially under the gauge group. Then, their mass splittings generate gaugino masses by one-loop diagrams involving gauge interactions. The latter lead to finite contributions given by [20]:

$$
m_{\lambda \lambda} \sim N_{s} \frac{\alpha}{2 \pi} \mu f\left(\frac{m_{s}}{\mu}\right),
$$

where $\mu$ is the supersymmetric mass and $m_{s}^{2}$ the squared mass splitting of those matter fields; $N_{s}$ denotes their multiplicity, $\alpha$ is the corresponding gauge coupling, and the function $f(x)$ is nearly constant for $x \gtrsim 2$ while it behaves as $x$ for $x \lesssim 1$. Thus, the gaugino masses are of the order of $(\alpha / 2 \pi) N_{s} \min \left(\mu, m_{s}\right)$.

It is easy to see that when $\mu$ is below the intermediate scale $\rho^{-1}$, the evaluation of the scalar masses (5.1) -(5.3) through the diagrams of Fig. 1 remains valid up to $\mathcal{O}\left(\mu / m_{3 / 2}\right)$ corrections. It follows that the gaugino masses are approximately one order of magnitude lower than the scalar masses if $\mu \gtrsim m_{s}$. Although this mechanism can give acceptable 
masses to charginos and neutralinos, provided that the Higgs supersymmetric parameter $\mu$ is large enough, gluino masses would require the Standard Model particle content to be extended by the presence of extra colour multiplets in vector-like representations such as triplets or leptoquarks. Of course, in this case, unification requires that the extra matter appears in complete $S U(5)$ representations, e.g. $(\mathbf{5}+\overline{\mathbf{5}})$ or $(\mathbf{1 0}+\overline{\mathbf{1 0}})$. Otherwise, in the absence of extra matter, this scenario leaves open the possibility of having light gluinos with masses of order $\left(\alpha_{3} / 2 \pi\right) m_{\text {top }}=\mathcal{O}(1) \mathrm{GeV}$.

To summarize, the mass spectrum we obtained in the observable sector originates from local supersymmetry breaking, with a gravitino mass $m_{3 / 2}$ at an intermediate scale defined by the size of the eleventh dimension of M-theory. All scalars then acquire masses of order $m_{3 / 2}^{2} / M_{p}$, while gaugino masses are of order $m_{3 / 2}^{3} / M_{p}^{2}$. This situation is again identical to the case where supersymmetry is broken in the heterotic string by gaugino condensation stabilized by a VEV of the antisymmetric tensor field strength [14, 15]. As we saw, the problem of having very light gauginos can be solved by means of gauge interactions involving extra fields and providing gaugino masses of order $(\alpha / 2 \pi) m_{3 / 2}^{2} / M_{p}$. Therefore, this scenario predicts a hierarchy of supersymmetric mass spectrum where scalars are much heavier than gauginos.

On the other hand, in the old analysis of gaugino condensation, based on the heterotic string tree-level effective supergravity, it was found that scalars remained massless at the one-loop order, because of the dilation properties of the Kähler potential [14]. In fact, it is easy to see that the term in the brackets of eq. (5.3) vanishes when the Kähler potential has for instance the no-scale $S U(1, n)$ form $K=-3 \ln \left(z+\bar{z}-|\varphi|^{2}\right)$ and $\varphi$ 's have zero VEVs. This can lead to an alternative scenario where gauginos, with masses of order $m_{3 / 2}^{3} / M_{p}^{2} \sim 1 \mathrm{TeV}$ (for $m_{3 / 2} \sim 10^{14} \mathrm{GeV}$ ), seed supersymmetry breaking in the rest of the observable sector by gauge interactions. Since in this case the corresponding diagrams are logarithmically divergent, all supersymmetric masses turn out to be of the same order 
of magnitude. However, this scenario is expected (and was explicitly shown [15]) to be unstable under higher-order loop corrections, as the dilation symmetry is in general broken at the quantum level.

\section{Conclusions and outlook}

In this paper we have presented a mechanism for supersymmetry breaking by compactification of M-theory to four dimensions. We argued that this mechanism describes ordinary gaugino condensation in the strong coupling regime of the heterotic string. It requires the eleventh dimension of M-theory to be large at an intermediate scale $\rho^{-1} \sim 10^{12} \mathrm{GeV}$, which fixes the mass of the gravitino as well as that of all fermionic partners of moduli that live on the effective five-dimensional bulk. Supersymmetry breaking is communicated to our observable world, which lives on the four-dimensional boundary, by gravitational interactions. A computation of the mass splittings generically provides a hierarchy of soft masses at the TeV scale $\left(\sim \rho^{-2} / M_{p}\right)$, with matter scalars much heavier than gauginos.

Consistency of this mechanism requires the vanishing of quadratic divergences, and hence the hierarchy of soft masses, to persist at higher loops. Indeed, by a preliminary inspection of higher-loop diagrams we expect this to be the case, in close analogy with the situation at finite temperature. On the other hand, this scenario seems to suffer from the usual cosmological problems, namely the cosmological constant that appears to be of the order of the intermediate scale $m_{3 / 2}^{4}$ and the problem of moduli that have masses at the $\mathrm{TeV}$ scale. Furthermore, since scalar masses are not necessarily universal they might induce flavour-changing neutral currents. Although we have not discussed in this work the question of dynamical determination of the intermediate scale $\rho$, we would like to point out that the required hierarchy $\rho M_{11} \sim 10^{4}$ is significantly smaller than the usual hierarchy $M_{G} / m_{\mathrm{SUSY}} \sim 10^{13}$.

The above results can be contrasted with those where supersymmetry breaking is in- 
duced by a large internal dimension $R$ in the heterotic string [17, 18]. Unlike the present situation, in the latter case there is also, on top of the gravitational sector, ordinary matter including the gauge vector multiplets living in the bulk of the large internal dimension. As a consequence the gravitino and gauginos get masses of the order of $R^{-1}$, and so the corresponding radius $R$ is required to have a value of order $1 \mathrm{TeV}^{-1}$. The seed of supersymmetry breaking is then communicated by gauge interactions from the gauginos to the scalar sector. As a result, universality of scalar masses is approximately maintained and there is no hierarchy among soft-breaking parameters. The cosmological constant generated at one loop is $\mathcal{O}\left(R^{-4}\right)$, much smaller than in the present mechanism. This also allows the dynamical determination of $R$ through the low-energy radiative breaking of weak interactions. However, to avoid large threshold corrections to gauge couplings, spoiling the validity of perturbation theory, one has to consider special models where these corrections do not appear.

We believe it is worth while to complete the present analysis with a more detailed computation of the mass spectrum and couplings (including trilineal soft terms) of the lowenergy effective field theory. In this work, we have used a particular $Z_{2}$ discrete symmetry to break supersymmetry by the radius of the eleventh dimension. One can thus ask the question of the existence of other possible discrete symmetries that can also be used, and, of their effects in the low-energy theory.] Nevertheless, the general features of the breaking will be maintained, provided that one uses the same radius of $S^{1}$ to break supersymmetry in compactifications of M-theory on $\mathrm{CY} \times S^{1} / Z_{2}$. The main reason is that the observable sector lives on the boundary and, thus, does not receive any mass splitting to lowest order. Supersymmetry breaking is then communicated by gravitational interactions and yields the same hierarchy in the mass spectrum as that we have found in this paper. The only possibility to avoid this hierarchy would be in models with special dilation properties of the

\footnotetext{
${ }^{2}$ See also the recent analysis of Ref. [21].
} 
Kähler potential. It would be interesting to study whether such models can be built using the particular features of the effective theory in the large radius limit discussed in section 5, and whether these models can escape some of the above-mentioned problems. Finally, it is an open question to find an M-theory formulation of this mechanism of supersymmetry breaking using for instance M(atrix)-theory.

\section{References}

[1] P. Hořava and E. Witten, Nucl. Phys. B460 (1996) 506 and B475 (1996) 94.

[2] E. Witten, Nucl. Phys. B471 (1996) 135.

[3] P. Hořava, Phys. Rev. D54 (1996) 7561.

[4] I. Antoniadis and M. Quirós, Phys. Lett. B392 (1997) 61.

[5] J. Scherk and J.H. Schwarz, Nucl. Phys. B153 (1979) 61; J. Scherk and J.H. Schwarz, Phys. Lett. B82 (1979) 60; E. Cremmer, J. Scherk and J.H. Schwarz, Phys. Lett. B84 (1979) 83; P. Fayet, Phys. Lett. B159 (1985) 121, Nucl. Phys. B263 (1986) 649.

[6] A.C. Cadavid, A. Ceresole, R. D’Auria and S. Ferrara, Phys. Lett. B357 (1995) 76; I. Antoniadis, S. Ferrara and T.R. Taylor, Nucl. Phys. B460 (1996) 489.

[7] T. Li, J.L. Lopez and D.V. Nanopoulos, e-print hep-ph/9702237.

[8] E. Cáceres, V.S. Kaplunovsky and I.M. Mandelberg, Nucl. Phys. B493 (1997) 73.

[9] J.-P. Derendinger, L.E. Ibáñez and H.P. Nilles, Phys. Lett. B155 (1985) 65; M. Dine, R. Rohm, N. Seiberg and E. Witten, Phys. Lett. B156 (1985) 55; C. Kounnas and M. Porrati, Phys. Lett. B191 (1987) 91. 
[10] G. Veneziano and S. Yankielowicz, Phys. Lett. B113 (1982) 231; T.R. Taylor, G. Veneziano and S. Yankielowicz, Nucl. Phys. B218 (1983) 493; T.R. Taylor, Phys. Lett. B164 (1985) 43.

[11] M.A. Shifman and A.I. Vainstein, Nucl. Phys. B277 (1986) 456 and B359 (1991) 571.

[12] C.P. Burgess, J.-P. Derendinger, F. Quevedo and M. Quirós, Ann. Phys. 250 (1996) 193.

[13] R. Rohm and E. Witten, Ann. Phys. 170 (1986) 454.

[14] Y.J. Ahn and J.D. Breit, Nucl. Phys. B273 (1986) 75; P. Binétruy, S. Dawson and I. Hinchliffe, Phys. Lett. B179 (1986) 262.

[15] J. Ellis, D.V. Nanopoulos, M. Quirós and F. Zwirner, Phys. Lett. B180 (1986) 83; J. Ellis, A.B. Lahanas, D.V. Nanopoulos, M. Quirós and F. Zwirner, Phys. Lett. B188 (1987) 408 .

[16] C. Kounnas and M. Porrati, Nucl. Phys. B310 (1988) 355; S. Ferrara, C. Kounnas, M. Porrati and F. Zwirner, Nucl. Phys. B318 (1989) 75; C. Kounnas and B. Rostand, Nucl. Phys. B341 (1990) 641.

[17] I. Antoniadis, Phys. Lett. B246 (1990) 377; Proc. PASCOS-91 Symposium, Boston 1991 (World Scientific, Singapore), p. 718.

[18] I. Antoniadis, C. Muñoz and M. Quirós, Nucl. Phys. B397 (1993) 515; I. Antoniadis and K. Benakli, Phys. Lett. B326 (1994) 69; I. Antoniadis, K. Benakli and M. Quirós, Phys. Lett. B331 (1994) 313; K. Benakli, Phys. Lett. B386 (1996) 106.

[19] T. Li, J.L. Lopez and D.V. Nanopoulos, e-print hep-ph/9704247.

[20] R. Barbieri, L. Girardello and A. Masiero, Phys. Lett. B127 (1983) 429.

[21] E. Dudas and C. Grojean, e-print hep-th/9704177. 


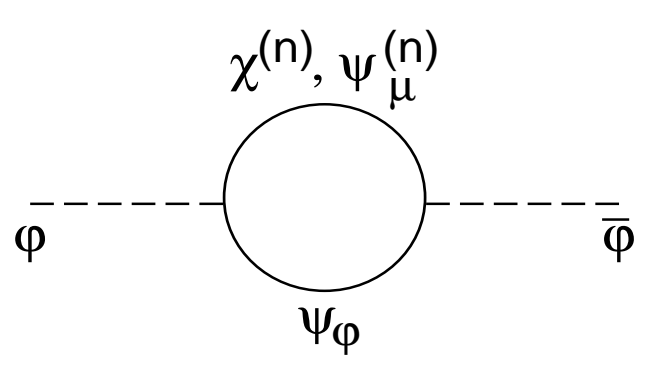

(a)

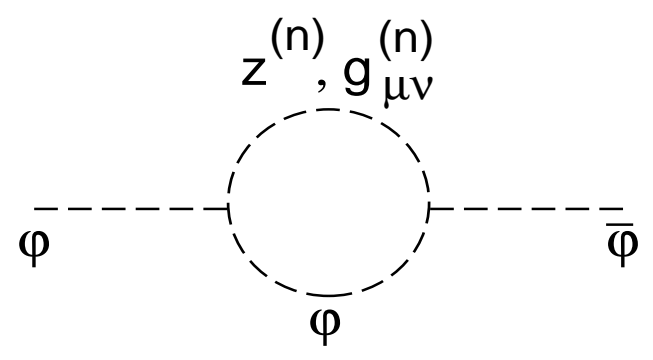

(c)

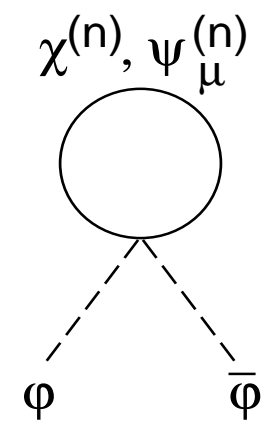

(b)

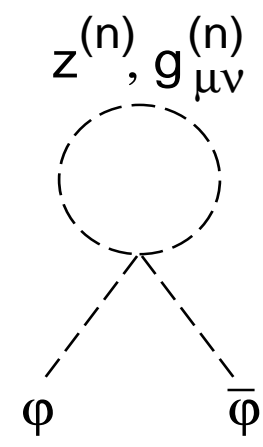

(d)

Figure 1: One-loop (gravitational) diagrams contributing to scalar masses. 


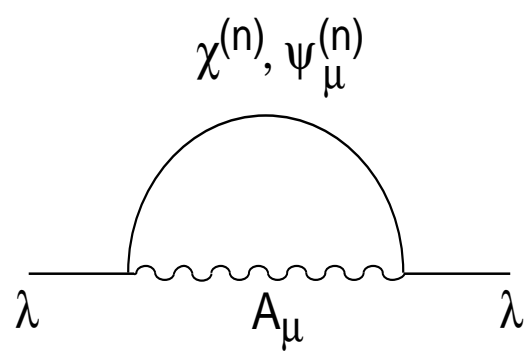

(a)

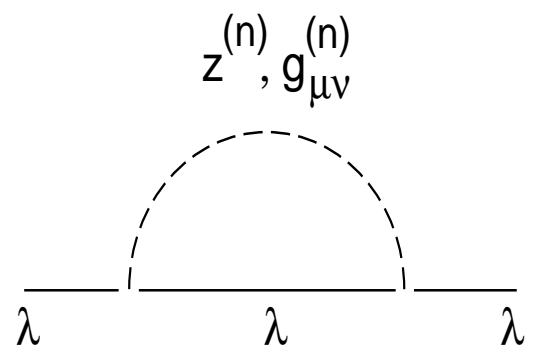

(c)

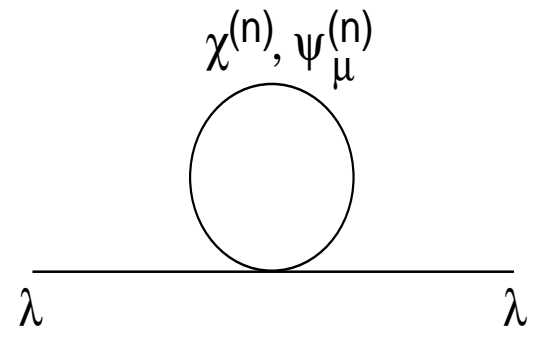

(b)

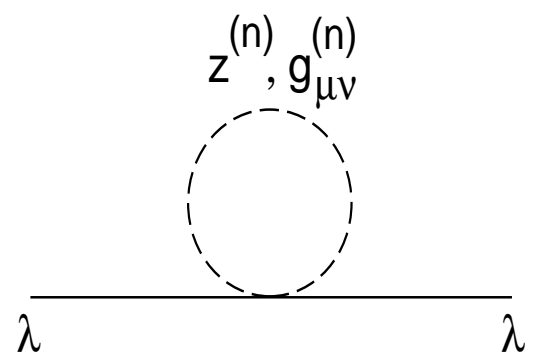

(d)

Figure 2: One-loop (gravitational) diagrams contributing to gaugino masses. 